\title{
A NEW PROOF OF A THEOREM OF DIRAC
}

\author{
William G. Brown
}

(received October 30,1964 )

In [1] Dirac has determined the structure of all 3-connected graphs which do not contain two independent (i. e., disjoint) circuits. We shall here provide a short proof of this theorem by applying Tutte's theory of 3-connected graphs [2].

For definitions of the terms used the reader is referred to [1]. We shall restrict our attention to simple graphs, i.e. graphs without multiple edges. $<k>$ will denote the complete $k$-graph. $\quad W_{k}$ will denote the $k$-wheel: a graph whose vertices are labelled $0,1, \ldots, k$, with edges $(0,1), \ldots,(0, k),(1,2)$, $(2,3), \ldots,(k-1, k),(k, 1) . \quad p^{K}$ will denote the graph with vertices $x_{1}, x_{2}, x_{3}, y_{1}, \ldots, y_{p}$ and edges $\left(x_{i}, y_{j}\right)$ $(i=1,2,3 ; j=1,2, \ldots, p)(p>1)$. We define $\mathrm{p}_{1}=\mathrm{p}^{\mathrm{K}}+\left(\mathrm{x}_{1}, \mathrm{x}_{2}\right)$, $\mathrm{p}_{2}=\mathrm{p}_{1}+\left(\mathrm{x}_{2}, \mathrm{x}_{3}\right), \mathrm{p}_{3}=\mathrm{K}_{2}+\left(\mathrm{x}_{3}, \mathrm{x}_{1}\right)$; the subscripts $\mathrm{p}$ may be suppressed. The class of graphs having two independent circuits will be denoted by $\Omega$.

The theorem of Tutte we require states that every simple 3-connected graph having more than 3 vertices is either a wheel or can be obtained from a wheel by a sequence of operations of the following two types:

I. inscribing a new edge;

II. replacing a vertex $x$ by two vertices $x^{\prime}, x^{\prime \prime}$ connected by an edge, such that every vertex formerly connected to $\mathbf{x}$ is connected to exactly one of $x^{\prime}, x^{\prime \prime}$, and such that each of $\mathbf{x}^{\prime}, \mathbf{x}^{\prime \prime}$ is connected to at least two of the se vertices.

Canad. Math. Bull. vol. 8, no. 4, June 1965 
We observe that any graph obtained from a graph in $\Omega$ by Operations I or II aIso lies in $\Omega$.

Dirac's result [1, pp. 186-193] is

THEOREM. The only 3 -connected (simple) graphs with at least 4 vertices and not in $\Omega$ are of the following types:

1) $w_{k}(k>3)$;

2) $\mathrm{p}^{\mathrm{K}}, \mathrm{p}^{\mathrm{K}}, \mathrm{p}^{\mathrm{K}}{ }^{\prime} \mathrm{p}^{\mathrm{K}} 3(\mathrm{p}>2)$;

3) ${ }_{2} \mathrm{~K}_{3}$;

4) $<5>$.

We first observe that none of the graphs listed lies in $\Omega$.

LEMMA 1. An application of I or II to $W_{k}(k>4)$ yields a graph in $\Omega$.

Proof:

a) Label the vertices so that an added edge is $(1, n)$ where $3 \leq \mathrm{n} \leq[(\mathrm{k}+1) / 2]$. Then two independent circuits are $((\overline{1}, 2, \overline{3}, \ldots, n))$ and $((0, n+1, n+2, \ldots, k))$.

b) The only vertex at which II can be applied is 0 . Suppose one of $0^{\prime}$, $0^{\prime \prime}$, say $0^{\prime}$, is connected to two consecutive vertices, which can be taken to be 1 and 2 . Then there must exist two vertices $\mathrm{p}$ and $\mathrm{q}$ connected to $0^{\prime \prime}$ and such that $2<\mathrm{p}<\mathrm{q}<\mathrm{k}$. Then two circuits are $\left(\left(1,0^{\prime}, 2\right)\right)$ and $\left(\left(\mathrm{p}, \mathrm{p}+1, \ldots, \mathrm{q}, \mathrm{0}^{\prime \prime}\right)\right)$. Otherwise the vertices can be so labelled that $0^{\prime \prime}$ is connected to all even vertices, and $0^{\prime}$ to all odd vertices. Two circuits are $\left(\left(1,0^{\prime}, 3,2\right)\right)$ and $\left(\left(4,0^{\prime \prime}, 6,5\right)\right)$ (here $k \geq 6$ ).

It follows from Lemma 1 and the theorem of Tutte that the only graphs other than the wheels that we need consider are those obtainable from $\mathrm{W}_{4}$ by a sequence of operations I and II. We note that $W_{4}$ is a ${ }_{2} K_{2}$. 
LEMMA 2. Any graph obtained by operation II from a $K, K_{1}, K_{2}$, or $K_{3}$ and which does not lie in $\Omega$, is a $K$ or a $\mathrm{K}_{1}$.

Proof: The only vertices where II can be applied are the $x^{\prime} s$, as the $y^{\prime} s$ have valency less than 4.

a) Let a $K$ be given. Label the vertices so that $x_{1}$ is replaced by $x_{1}^{\prime}$ connected to $y_{1}, y_{2}, \ldots$, and $x_{1}^{\prime \prime}$ connected to $y_{3}, y_{4}, \ldots ;$ (p must be greater than 3 ). Then two circuits are $\left(\left(x_{1}^{\prime}, y_{1}, x_{2}, y_{2}\right)\right)$ and $\left(\left(x_{1}^{\prime \prime}, y_{3}, x_{3}, y_{4}\right)\right)$.

b) Let a $K_{1}$ be given. Applying $I I$ at $x_{3}$ yields a graph in $\Omega$ by the same argument as in a). Label the vertices so that $x_{1}$ is replaced by $x_{1}^{\prime}$ connected to $y_{1}, y_{2}, \ldots$ and $x_{1}^{\prime i}$ connected to $x_{2}, y_{3}, \ldots$; (here $p>2$ ). Then two circuits are $\left(\left(x_{1}^{\prime}, y_{1}, x_{3}, y_{2}\right)\right)$ and $\left(\left(x_{1}^{\prime \prime}, x_{2}, y_{3}\right)\right)$.

c) Let a $\mathrm{K}_{2}$ or $\mathrm{K}_{3}$ be given. When $\mathrm{p}>2$, the only type of application of II not considered above is where $x_{2}$ is replaced by $x_{2}^{\prime}$ connected to $x_{1}$ and $x_{3}$ only, and $x_{2}^{\prime \prime}$ connected to $y_{1}, \ldots y_{p^{\prime}}$ It can be seen by renaming $x_{3}, x_{2}^{\prime \prime}$ and $x_{2}^{\prime}$ respectively $x_{2}, x_{3}$, and $y_{p+1}$ that the resulting graph is a $\mathrm{K}$ or $\mathrm{K}_{1}$.

d) One further case remains when $p=2$. (I am indebted to the referee for drawing this to my attention.) Given a $2^{K_{2}}$ or $2 K_{3}$, let $x_{2}$ be replaced by $x_{2}^{\prime}$ connected to $x_{1}$ and $y_{2}$, and $x_{2}^{\prime \prime}$ connected to $x_{3}$ and $y_{2}$. Two circuits are $\left(\left(x_{1}, x_{2}^{\prime}, y_{2}\right)\right)$ and $\left(\left(x_{2}^{\prime \prime}, y_{1}, x_{3}\right)\right)$. 
LEMMLA 3. Any graph not in $\Omega$ obtained by operation I from a 3-connected $\mathrm{K}, \mathrm{p}_{1}, \mathrm{p}_{2}$, or $\mathrm{p}_{3}$ is
a) a $2^{K_{3}}$ or $\langle 5\rangle$ if $p=2$;
b) a $\mathrm{p}_{1}, \mathrm{p}_{2}$, or $\mathrm{p}_{3}$ if $\mathrm{p}>2$.

Proof: (We observe that $2^{K}, 2_{1}^{K}$ are not 3 -connected.) The lemma is obvious for a new edge connecting two $x^{\prime} s$. We consider the case where the new edge connects two $y^{\prime} s$ and assume the vertices labelled so that the new edge is $\left(y_{1}, y_{2}\right)$.

a) Let $p=2 . K_{2}$ yields a $K_{3}$; this can be seen by relabelling $x_{1}, x_{3}, y_{1}, y_{2}$ as $y_{1}, y_{2}, x_{1}, x_{3}$ respectively. $\mathrm{K}_{3}$ yields a $<5>$.

b) Let $p=3$. Clearly $K$ yields a $K_{1}$, (interchange $x^{\prime} s$ and $\left.y^{\prime} s\right)$. But $K_{1}$ yields a graph in $\Omega$ having circuits $\left(\left(x_{1}, x_{2}, y_{3}\right)\right)$ and $\left(\left(y_{1}, y_{2}, x_{3}\right)\right)$. Thus $K_{2}$ and $K_{3}$ also yield graphs in $\Omega$.

c) Let $p>3$. Then in all cases the circuits $\left(\left(x_{1}, y_{1}, y_{2}\right)\right)$ and $\left(\left(x_{2}, y_{3}, x_{3}, y_{4}\right)\right)$ are present.

To complete the proof of the theorem we need only remark that an operation II on $\langle 5\rangle$ (and all such operations are equivalent) yields a graph in $\Omega$. Thus, for $p>2$, operations $I$ on $\mathrm{p}^{\mathrm{K}} \mathrm{p}^{\mathrm{K}}{ }^{\prime} \mathrm{p}^{\mathrm{K}} \mathrm{2}^{\prime} \mathrm{p}_{3}$ yield only $\mathrm{p}_{1} \mathrm{p}^{\mathrm{K}} \mathrm{p}^{\prime}$ or $\mathrm{p}_{3}$ not in $\Omega$; and operations II on $\mathrm{p}^{\mathrm{K}} \mathrm{p}_{1} \mathrm{~K}_{\mathrm{p}} \mathrm{K}_{2} \mathrm{p}_{3}$ yield only $\mathrm{p}_{+1} \mathrm{~K}$ and $p+1 K_{1}$ not in $\Omega$. The proof follows by induction on $p$. 


\section{REFERENCES}

1. G. A. Dirac, Some results concerning the structure of graphs, Canad. Math. Bull. 6 (1963) 183-210.

2. W. T. Tutte, A theory of 3-connected graphs, Proc.

Koninkl. Akad. Wetenschappen A 64 (1961) 441-455.

University of British Columbia 